\title{
The ergonomics approach for thin film transistor-liquid crystal display manufacturing process
}

\author{
Chih-Wei Lu ${ }^{\mathrm{a},{ }^{*}}$, Chia-Chun Yao ${ }^{\mathrm{b}}$, Chein-Wen Kuo ${ }^{\mathrm{b}}$ \\ andustrial and systems Engineering Department, Chung Yuan Christian University,200, Chung Pei Rd., \\ Chung Li, Taiwan 32023, R.O.C. \\ ${ }^{\mathrm{b}}$ Graduate Institute of Occupational Safety and Heath, Kaohsiung Medical University then University, Kaohsiung \\ City, 80722, Taiwan, R.O.C.
}

\begin{abstract}
The thin film transistor-liquid crystal display (TFT-LCD) has been used all over the world. Although the manufacture process of TFT-LCD was highly automated, employees are hired to do manual job in module assembly process. The operators may have high risk of musculoskeletal disorders because of the long work hours and the repetitive activities in an unfitted work station. The tools of this study were questionnaire, checklist and to evaluate the work place design. The result shows that the participants reported high musculoskeletal disorder symptoms in shoulder $(59.8 \%)$, neck $(49.5 \%)$, wrist $(39.5 \%)$, and upper back (30.6\%). And, to reduce the ergonomic risk factors, revising the height of the work benches, chairs and redesigning the truck to decrease the chance of unsuitable positions were recommended and to reduce other ergonomics hazards and seta good human machine interface and appropriate job design.
\end{abstract}

Keywords: TFT-LCD, manufacturing process, musculoskeletal disorder, risk factor,

\section{Introduction}

The thin film transistor-liquid crystal display (TFT-LCD) has been used all over the world. And, the TFT-LCD industry has been growing rapidly and become one of the mainstream industries in Taiwan. Although the manufacture process of TFT-LCD was highly automated, employees are hired to do manual job in module assembly process. The operators may have high risk of musculoskeletal disorders because of the long work hours and the repetitive activities in an unfitted work station. The aim of the study was to evaluate the ergonomics hazards in the module assembly process in a TFTLCD factory.
The three TFT-LCD manufacturing processes were: 1) up-process (Array): take thin film transistor on glass; 2) middle-process (Cell) : using the glass as base to combine color filter, then inject liquid crystal inside; 3) end-process (Module): put all parts (glass, back-light, cell, circuit board..etc) together on a assembling line. The assembling manufacture processes were the target of this study. The module assembling processes were dynamic and heavy duty jobs. Operators have done repetitive motions during a long time. The muscles would be damaged, if muscle constricted repetitive and unnatural posture during long time, named cumulative trauma disorder (CTD) [1][2][3][4]. Moreover, operators have worked in clean room where workers needed to ware particle-

\footnotetext{
${ }^{*}$ Corresponding author. E-mail: chwelu@cycu.edu.tw the study has funded by National Science Council (NSC99-2221-E033070-My3 ) and Chung Yuan Christian University (CYCU-EESC-9901).
}

1051-9815/12/\$27.50 @ 2012 - IOS Press and the authors. All rights reserved 
free clothes and mask. Both would make operators' body temperature high and muscle fatigue, then, raise the risk of musculoskeletal disorder [5].

\section{Methods}

This was a cross-section study and operators who worked in module plants were participated. The step or procedure of this study were: 1) using questionnaire to survey the musculoskeletal disorder (MSDS) symptoms, 2) using job analysis and Baseline Risk Identification of Ergonomic Factor (BRIEF) to evaluate the hazards, 3 ) evaluating the work place design and 4) using a biomechanical model to measure the force and posture of the musculoskeletal system of the worker.

\subsection{Questionnaire r survey}

The questionnaire was used to evaluate the musculoskeletal disorder symptoms from operators. The questionnaire was revised from the Nordic Musculoskeletal Questionnaire NMQ [6].

\subsection{Job analysis and observation}

The goal of the job observation and analysis was to analyze the manufacturing processes into small piece, to record them, to describe each process and the characteristics of the motion of body. Then, by using ergonomics checklist the investigator could find the potential risks during the motion of operators.

The ergonomics checklist named BRIEF (Basic Risk Index of Ergonomics Factor) was used in the study. This checklist has been used for ergonomics risk factor investigation in industrial side couple years [7].

\subsection{In-plant work environment measurement}

The in-plant work environment measurement could let the investigator got the lay-out of factories and size of work machines. Based on the ergonomics principal, anthropometry data of operators and measurement of work environment, the investigator could evaluate whether there was potential ergonomics or musculoskeletal disorder risk in the factory or not.

\subsection{Biomechanics model analysis}

Biomechanics model was a tool to evaluate the force inside the body. The lifting Evaluation software V1.0 was used for measure the compress and shear force working on the L5/S1 spine disc [7]. The test of software listed the number of shear stress, compress force one the disk and allow limitation (AL), maximum permit limitation (MPL) [8]. The results of test were shown on three types:

(1)The disc stress was less than $3400 \mathrm{~N}$ ( $\mathrm{AL}$ ) : It was accepted loading, green light and smile human face figure shown on the screen.

(2)The disc stress was less than 6400N (MPL), but larger than $3400(\mathrm{AL})$ : It was danger loading, yellow light and angry face figure on the screen.

(3)The disc stress was larger than 6400N (MPL) : it was very danger loading, red light and crying face figure on the screen.

\section{Results}

The result shows that the participants reported high musculoskeletal disorder symptoms. The result of the Baseline Risk Identification of Ergonomic Factor (BRIEF) analysis was similar to those of the questionnaire but was higher as compared to those of the questionnaire. And, the number of force has loaded on L5/S1 spine disc.

\subsection{Questionnaire r survey}

The questionnaires were passed to the operators who worked in the module plants of TFT-LCD manufacture. The 281 workers participated and 70 $(24.9 \%)$ were male and $211(75.1 \%)$ were female. They were $26.8 \quad(\mathrm{SD}=4.6)$ years old, $57.4 \mathrm{~kg}$ $(\mathrm{SD}=13.2)$ weight, $161.8 \quad(\mathrm{SD}=7.9)$ height, has worked 26.1 months ( $S D=20.7)$.Most people have gotten high school degree. About exercise habit, 58 $(20.6 \%)$ were Yes; 223(79.4\%) The participants reported high musculoskeletal disorder symptoms in shoulder (59.8\%), neck (49.5\%), wrist (39.5\%), and upper back $(30.6 \%)$. There was statistical significant difference of the rate musculoskeletal disorder distribution between the months of work experience. 


\subsection{Job analysis and observation}

Job observation was always in coordinate with on-side hardware size measurement to discover musculoskeletal disorder symptoms. The BRIEF checklist was used to evaluate 135 operators in module manufacturing process. The height rates of potential ergonomics risk happened in neck (100\%), back $(83.0 \%)$, right shoulder $(55.5 \%)$, left shoulder $(48.8 \%)$, right hand and right wrist $(35.6 \%)$ and low rates happened in left hand wrist (18.5\%) and leg $(5.9 \%)$

\subsection{In-plant work environment measurement}

Work environment was a key part of ergonomics hazard analysis. Three types of data were measured: 1) the size of in-plant lay-out, hardware and work station; 2) the weights of goods or parts of lifting $\&$ carrying processes; 3 ) the anthropometry data of operators. The heights of work benches were from 90 to $100 \mathrm{~cm}$.

\subsection{Biomechanics model analysis}

Based on the in-plat observation, the three processes (cell open and transformation, electric static disk moving and aging goods moving) have been chosen for biomechanics analysis.

In the results of biomechanical analysis, only one operator working in the "AGING" area suffered a compressive force on the lumber disc at L5/S1 was more than $3400 \mathrm{~N}$, which was over the lifting limitation of the USA National Institute of Occupational Safety and health.

\section{Discussion}

In this study, 73\% people have reported musculoskeletal disorder. A study done by IOSH of Taiwan for manufacturing industries shows $63.3 \%$ people have reported musculoskeletal disorder [9]. Its musculoskeletal disorder rate is less than this study. The segments of height musculoskeletal disorder rate were shoulder (59.8\%), neck (49.5\%), wrist (39.5\%), up-back (30.6\%) and Low-back (26.3\%) By checklist investigation, the height musculoskeletal disorder risk segments were neck (100\%), back $(83.0 \%)$, right shoulder (55.5\%), left shoulder $48.8 \%$ ), right wrist $(36.5 \%)$. It was similar with Lu' $\mathrm{s}$ who has used 3 checklists to investigate the musculoskeletal disorder for semiconductor industrial factories and found the height risk happened in the segment of shoulder and wrist [9].

\section{Conclusions}

The result shows that the participants reported high musculoskeletal disorder symptoms in shoulder (59.8\%), neck (49.5\%), wrist (39.5\%), and upper back (30.6\%). The result of the Baseline Risk Identification of Ergonomic Factor (BRIEF) analysis was similar to those of the questionnaire but was higher as compared to those of the questionnaire.

The heights of work benches were $90-100 \mathrm{~cm}$. In the results of biomechanical analysis, only one operator working in the "AGING" area suffered a compressive force on the lumber disc at L5/S1 was more than $3400 \mathrm{~N}$, which was over the lifting limitation of the USA National Institute of Occupational Safety and health[10]. The study indicated that the prevalence of the musculoskeletal symptoms were very high among participants in the module assembly process. To reduce the ergonomic risk factors, revising the height of the work benches, chairs and redesigning the truck to decrease the chance of unsuitable positions were recommended. To reduce other ergonomics hazards and seta good human machine interface and appropriate job design, we need to do more studies and pay more attention on the new industry.

\section{Acknowledge}

Thanks for the help from the operators at the TFTLCD factories in Taiwan. Also, Thanks for National Science Council (NSC 99-2221-E033-070-My3) and the Chung Yuan Christian University (CYCU-EECS9901).

\section{References}

[1] Institute of occupational safety and health. Manuduction of Ergonomics Musculoskeletal Disorders Prevention. Taipei : The Institute; 2001 IOSH-t042

[2]K-W Lin, W-Y Yeh, C-CChu, C-Y Chen. Survey of Cumulatively Work-related Disorders in the Semiconductor Industry. 
Semiconductor Industry. Institute of occupational safety and health quarterly publication $1997 ; 5$ ( 1 ) : 1-14

[3]Institute of occupational safety and health. Shoulder and Neck Disorders in Semiconductor Industry workers. Taipei : The Institute; 1998 IOSH87-M322 .

[4] Y-H Lin, W-Y Yeh. Musculoskeletal Disorders of workers in the Semiconductor Industry. IOSH wrap-up1999; 4

[5] C-Y Chen, Y-H Lin. Discussion of Ergonomics Potential Hazards in the Semiconductor Industry. IOSH wrap-up1999; 24 .

[6] I. Kuorinka, B. Jonsson, A. Kilborm, H. Vinterberg, BieringSorensen,F. Standardised Noric Questionnaire for the analysis of musculoskeletal symptoms,. Applied Ergonomics 1987 ; $18(3): 233-237$
[7] C. Lu, C. Chang, W. Yeh, Y. Lin, C. Chen. Application of Ergonomic Checklists in the Semiconductor Industry. Institute of occupational safety and health, quarterly publication 1999 ;

$4: 423-44$

[8] Institute of occupational safety and health. Program of Lowback Loading on Carry Material. Taipei : The Institute; 2000.

[9] Institute of occupational safety and health. Survey of Safety and Health cognition in work environment. Taipei : The Institute; 2001. IOSH90-H304

[10] D.Chaffin, B. Andersson, Occupational Biomechanics 2nd ed. Wiley-interscience Publisher; 1991; 264-301 